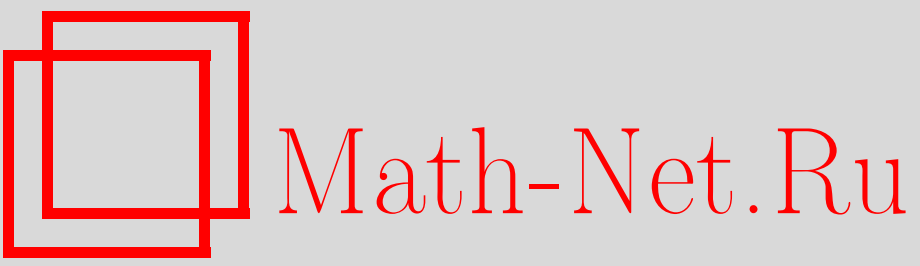

Ю. В. Брежнев, Об уравнениях Дубровина для конечнозонных операторов, УМH, 2002, том 57, выпуск 2, 191-192

DOI: https://doi.org/10.4213/rm503

Использование Общероссийского математического портала Math-Net.Ru подразумевает, что вы прочитали и согласны с пользовательским соглашением

http://www.mathnet.ru/rus/agreement

Параметры загрузки:

IP: 54.198 .67 .100

26 апреля 2023 г., 15:34:02

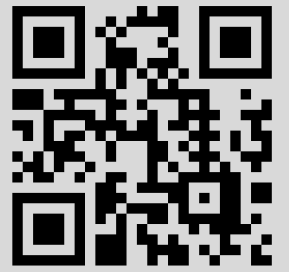




\title{
ОБ УРАВНЕНИЯХ ДУБРОВИНА ДЛЯ КОНЕЧНОЗОННЫХ ОПЕРАТОРОВ
}

\author{
Ю. В. БРЕЖНЕВ
}

До недавнего времени не поднимался вопрос: как перенести построения, связанные с уравнениями Дубровина (УД) в теории конечнозонных потенциалов оператора Шрёдингера [1], на произволшные спектралњные задачи? Имеются в виду уравнения на нули $\Psi$-функции и формулы следов. Хотя физическая интерпретация этих объектов как аналогов данных рассеяния в обобщениях не столь очевидна, либо вовсе отсутствует, упомянутая проблема имеет самостоятельную ценность, хотя бы по той причине, что известные уравнения Новикова, возникающие в общей теории конечнозонного интегрирования, являются (по всей видимости в самом общем случае) вполне интегрируемыми конечномерными динамическими системами, а сведение УД к задаче обращения Якоби реализует их интегрируемость по Лиувиллю. Добавим, что если алгебраическая кривая накрывает эллиптические кривые, то по формулам следов предъявляются решения в эллиптических функциях, собственно новые конечнозонные потенциалы и различные приложения к нелинейным уравнениям в частных производных.

В заметке [2] было обнаружено универсальное свойство конечнозонных потенциалов: они образуют класс, для которого спектральная задача интегрируется в квадратурах. Там же показано, как получать все ингредиенты прямой спектральной задачи: $\Psi$-формулу, алгебраическую кривую, уравнения Новикова и их интегралы. Если, коль скоро известна $\Psi$, естественно ожидать, что уравнения на ее нули $\gamma_{k}(x)$ должны выписьваться на основании элементарных соображений. Это имеет место быть, и мы показываем, как алгоритмически решается проблема с появлением сопутствующих моментов: формул следов и преобразования Абеля. Мы не обсуждаем здесь отдельньй вопрос о точном соответствии между алгебраической кривой и дивизором нулей $\left\{\gamma_{k}\right\}$ с одной стороны и краевыми условиями типа Дирихле $\Psi\left(x_{\mathrm{o}}\right)=\Psi\left(x_{\mathrm{o}}+\Omega\right)=0$ с другой в контексте восстановления потенциала по этим данньм как данным рассеяния на интервале для произвольных спектральных задач. Совокупность $\left\{\gamma_{k}\right\}$ рассматривается как отправной пункт. Продемонстрируем идеологию на примере скалярного операторного $\lambda$-пучка 3 -го порядка

$$
\Psi^{\prime \prime \prime}+u(\lambda ; x) \Psi^{\prime}+v(\lambda ; x) \Psi=0,
$$

где $u, v$ - рационалшные функции по $\lambda$ с полюсами, не зависящими от $x$. Условимся называть их потенциалом $[U]$. Пусть он конечнозонный. Тем самым $\Psi$-функция, возможно, станет многоточечной функцией Бейкера-Ахиезера [3]. Операторньй пучок, коммутирующий с (1) (т.е. второе уравнение на общую собственную функцию), в общем случае имеет вид:

$$
A(\lambda ; x) \Psi^{\prime \prime}+B(\lambda ; x) \Psi^{\prime}+C(\lambda ; x) \Psi=\mu \Psi, \quad C=\frac{2}{3} u A-\frac{1}{3} A^{\prime \prime}-B^{\prime},
$$

где $A$ и $B$ - полиномы по $\lambda$ и дифференциальные полиномы от [U]. Следуя [2], получаем алгебраическую кривую $W(\mu, \lambda)=0$ и два необходимых представления для $\Psi$-функции

$$
\begin{gathered}
\frac{\Psi^{\prime}}{\Psi}=\frac{\left(A^{\prime}+B\right)(\mu-C)+A C^{\prime}-v A^{2}}{A \mu-F}=-3 \frac{\left(\mu^{2}+Q\right) A^{2}+A F \mu+F^{2}}{A \Pi}+\frac{A^{\prime}+B}{A}, \\
-3 F=A A^{\prime \prime}+u A^{2}+3 B\left(A^{\prime}+B\right), \\
\Pi(\lambda ; x)=\left(3 v-u^{\prime}\right) A^{3}+\left(2 A^{\prime \prime \prime}+3 B^{\prime \prime}+3 u B+2 u A^{\prime}\right) A^{2}+3 A B\left(A^{\prime \prime}+B^{\prime}\right)+3 B^{2}\left(A^{\prime}+B\right) .
\end{gathered}
$$

Второе выражение для $\Psi$ в $(2)$ получено обращением в полином первого, как рациональной функции по $\mu$, с помощью уравнения кривой: $W(\mu, \lambda) \equiv \mu^{3}+Q(\lambda) \mu+R(\lambda)=0$. Полная совокупность нулей $\left\{\gamma_{k}\right\}$ на всех листах римановой поверхности $W(\mu, \lambda)=0$ определяется полюсами $\Psi^{\prime} / \Psi$ и факторизует знаменатель $\Pi=a \cdot\left(\lambda-\gamma_{1}\right) \cdots\left(\lambda-\gamma_{n}\right)$. В свою очередь, знаменатель первой из формул (2) определяет вторую координату (номер листа) на кривой нуля $\Psi$-функции: $\mu_{k}=\mu\left(\gamma_{k}(x)\right)$. Имеем отсюда, что $F(\lambda ; x) \rightarrow \mu_{k}(x) A\left(\gamma_{k} ; x\right)$, когда $\lambda \rightarrow \gamma_{k}$. Используя этот факт и осуществляя во 2-й формуле (2) предел $\lambda \rightarrow \gamma_{k}$, получаем аналоги УД:

$$
\gamma_{k}^{\prime}=3 A\left(\gamma_{k} ; x\right) \frac{3 \mu_{k}^{2}+Q\left(\gamma_{k}\right)}{a \prod_{j \neq k}\left(\gamma_{k}-\gamma_{j}\right)}, \mu_{k}^{\prime}=-3 A\left(\gamma_{k} ; x\right) \frac{Q^{\prime}\left(\gamma_{k}\right) \mu_{k}+R^{\prime}\left(\gamma_{k}\right)}{a \prod_{j \neq k}\left(\gamma_{k}-\gamma_{j}\right)}, \mu_{k}=\frac{F\left(\gamma_{k} ; x\right)}{A\left(\gamma_{k} ; x\right)}
$$

Работа выполнена при финансовой поддержке Российского фонда фундаментальных исследований (грант № 00-01-00782) и Королевского Общества Великобритании. 
До этого места мы ничем не ограничивались, поэтому уравнения (3) справедливы для любого операторного пучка (1), а способ вьвода без изменений переносится на высшие порядки. Первая система уравнений в (3) недавно появилась в [4] на примере спектральной задачи уравнения Буссинеска (не считая опечатки в $t$-группе УД (5.34) на с. 852). Однако мотивировки, доказательства и примеры не приведены. В то же время методика их получения требует лишь приведенных простых рассуждений. Отметим еще два принципиальных момента, не затрагиваемые в [4]. 1) УД следует привести к автономному виду. В форме же (3) они содержат потенциал в $A$-функции. 2) Потенциал $[U]$ выражается через $\Psi$-функцию (т.е. через $\Theta$ ). Опираясь на это, сформулируем вопрос: для каких $\lambda$-пучков конечнозонный потенчиал однозначно восстанавливается по координатам нулей $\Psi$ ? будет ли он абелевой функцией на якобиане кривой? Оператор Шрёдинге$\mathrm{pa}$, не считая родственных модификаций, является единственным известным примером, где этот вопрос решается главной формулой следов, содержащей $\sum \gamma_{k}$. Но произвольная абелева функция есть симметрическая комбинация верхних пределов абелевых интегралов в задаче обращения Якоби. Координаты $\left(\gamma_{k}, \mu_{k}\right)$ становятся равноправными, и, возможно, обе войдут в тождества следов. Этим обусловлено появление второй группы уравнений в (3). Нетрудно привести примеры, когда разрешение пункта 1) далеко не очевидно (и даже невозможно), и общий рецепт решения сформулированной проблемы пока не известен, рассмотрим показательньй пример:

$$
\begin{gathered}
\Psi^{\prime \prime \prime}+u(x) \Psi^{\prime}=\lambda \Psi, \quad\left(3 u^{\prime}-9 \lambda\right) \Psi^{\prime \prime}+\left(u^{2}-u^{\prime \prime}+\alpha\right) \Psi^{\prime}-6 \lambda u \Psi=\mu \Psi, \\
W(\mu, \lambda): \mu^{3}+\left(27 \alpha \lambda^{2}+E_{2}\right) \mu+729 \lambda^{5}+81 E_{1} \lambda^{3}+E_{3} \lambda=0, \quad \text { род } g=4 .
\end{gathered}
$$

Максимальное, что извлекается из факторизации полинома П, так это формула $2 u^{\prime}=3 \sum \gamma_{k}$. Но, комбинируя ее с выражением для интегралов $E_{1,3}\left(u, u^{\prime}, \ldots, u^{(\mathrm{iv})}\right)$ и формулами (3), получаем, как ответ на вопрос, три варианта главной формулы следов соответственно:

$u=-\frac{3}{2 \alpha}\left\{E_{1}+\sum_{k=1}^{4}\left(\gamma_{k}^{\prime \prime \prime}+\frac{15}{2} \gamma_{k}^{2}\right)\right\}, u=\frac{1}{6} \sum_{k=1}^{4} \gamma_{k} \mu_{k} \frac{\sum_{j=1}^{4} \gamma_{j}-2 \gamma_{k}}{\prod_{j \neq k}\left(\gamma_{k}-\gamma_{j}\right)}, u=\frac{E_{3}+3^{6} \gamma_{1} \gamma_{2} \gamma_{3} \gamma_{4}}{6 E_{1}}$.

Интегральная форма уравнений (3) в виде задачи обращения Якоби и базис голоморфных дифоференциалов для тригональной негиперэллиптической кривой (4) имеют вид:

$$
\left\{\begin{array}{l}
\sum_{k=1}^{4} \int^{\left(\gamma_{k}, \mu_{k}\right)} \frac{d \lambda}{3 \mu^{2}+Q(\lambda)}=a_{1}, \sum_{k=1}^{4} \int^{\left(\gamma_{k}, \mu_{k}\right)} \frac{\lambda d \lambda}{3 \mu^{2}+Q(\lambda)}=a_{2}, \\
\sum_{k=1}^{4} \int^{\left(\gamma_{k}, \mu_{k}\right)} \frac{\mu d \lambda}{3 \mu^{2}+Q(\lambda)}=a_{3}, \sum_{k=1}^{4} \int^{\left(\gamma_{k}, \mu_{k}\right)} \frac{\lambda^{2} d \lambda}{3 \mu^{2}+Q(\lambda)}=a_{4}-\frac{1}{81} x,
\end{array} Q(\lambda)=27 \alpha \lambda^{2}+E_{2} .\right.
$$

Голоморфные абелевы дифференциалы для любой кривой $W(\mu, \lambda)=0$ можно записать в каноническом виде $d \omega=P(\mu, \lambda) W_{\mu}^{-1} d \lambda[5 ; \S 39]$, однако нельзя утверждать, что к ним всегда приводят УД. Достаточно контрпримера $\Psi^{\prime \prime}=\lambda u(x) \Psi$, когда прямые аналоги ни УД, ни формул следов не имеют места. Но в этом примере $\Psi$ не является классической функцией Бейкера-Ахиезера [3], хотя коммутирующий оператор, кривая и $\Psi$-форормула предъявляются.

\section{СПИСОК ЛИТЕРАТУРЫ}

[1] Б. А. Дубровин // Функц. анализ и его прил. 1975. Т. 9. № 3. С. 41-51. [2] Н. В. Устинов, Ю. В. Брежнев // УМН. (в печати). [3] И. М. Кричевер // УМН. 1977. Т. 32. № 6. C. 183-208. [4] R. Dickson, F. Gesztesy, K. Unterkofler // Rev. Math. Phys. 1999. V. 11. P. 823-879. [5] Н. Г. Чеботарев. Теория алгебраических функций. М.-Л.: Гостехиздат, 1948. 